\title{
The Sensitivity of Cu for Electrochemical Carbon Dioxide Reduction to Hydrocarbons as Revealed by High Throughput Experiments
}

\author{
Yungchieh Lai ${ }^{a}$, Ryan J.R. Jones ${ }^{a}$, Yu Wang $^{\mathrm{a}}$, Lan Zhou ${ }^{\mathrm{a}}$, Matthias H. Richter ${ }^{\mathrm{a}}$, and John Gregoire ${ }^{\mathrm{a}, \mathrm{b},{ }^{*}}$
}

a. Joint Center for Artificial Photosynthesis, California Institute of Technology, Pasadena, CA 91125

b. Division of Engineering and Applied Science, California Institute of Technology, Pasadena, CA 91125

* gregoire@caltech.edu

\begin{abstract}
:
Electrochemical $\mathrm{CO}_{2}$ reduction to valuable products is a centerpiece of future energy technologies that relies on identificaiton of new catalysts. We present accelerated screening of $\mathrm{Cu}$ bimetallic alloys, revealing remarkable sensitivity to alloy concentration that indicates the segregation of alloying elements to critical sites for hydrocarbon formation.
\end{abstract}

The promise of electrochemical $\mathrm{CO}_{2}$ reduction to valuable products has prompted substantial research in selective catalysis of the many-electron reduction of $\mathrm{CO}_{2}$ to products such as light hydrocarbons. ${ }^{1}$ However, most metal electrocatalysts have been shown to exclusively form the $2 \mathrm{e}^{-}$products $\mathrm{CO}$ and formate (e.g. Au, Ag, In, and Sn) and/or catalyze the competing hydrogen evolution reaction (HER) (e.g. $\mathrm{Fe}, \mathrm{Ni}, \mathrm{Pt}$, and $\mathrm{Ti}$ ). ${ }^{2} \mathrm{Cu}$ is the only known elemental catalyst capable of reducing $\mathrm{CO}_{2}$ to hydrocarbons and alcohols with appreciable Faradaic efficiency (FE) but suffers from poor selectivity to any single product and high overpotentials for generating the highly reduced products. ${ }^{2-4}$ The uniqueness of $\mathrm{Cu}$ has been understood to be due to its moderate binding energy of CO and other intermediates. ${ }^{5,6}$ Alloying with a secondary metal is a widely-adopted strategy to alter a catalyst's adsorption energetics for reactants and intermediates, and some copper-based bimetallic catalysts have been evaluated for altering the $\mathrm{CO}_{2} \mathrm{RR}$ selectivity. ${ }^{7}$ While improved selectivity for $2 \mathrm{e}^{-}$products has been achieved, success in increasing FE for higher order products has been more limited, as discussed further below. ${ }^{8-22}$ Comparison among reported Cu-alloy systems is limited by the variation in catalyst syntheses and morphologies in these studies, motivating the systematic study of different alloying elements and alloying concentrations in the present work.

Exploration of $\mathrm{CO}_{2} \mathrm{RR}$ electrocatalysts is hampered by the dearth of accelerated screening systems, which are challenging to realize given the sensitivity of product distribution to the electrochemical environment and the breadth of possible reaction products. The ideal characterization of a given catalyst is quantitative measurement of the activity and product distribution, as a function of time, and for a large range of overpotentials since product distributions vary with aging of the operational catalyst as well as the operating potential. This combination of desired measurement attributes is incompatible with traditional experiments, perhaps most notably due to the duration of quantitative product distribution measurements via chromatography or nuclear magnetic resonance, severely limiting the measurement frequency to observe variations in product distribution as a function of time and/or overpotential. Through principled design of an accelerated screening system, we demonstrate quantitative Faradaic efficiency (FE) measurement of $\mathrm{H}_{2}, \mathrm{CH}_{4}$, and $\mathrm{C}_{2} \mathrm{H}_{4}$ during cyclic voltammetry $(\mathrm{CV})$ experiments with a measurement interval of $12 \mathrm{~s}$, enabling rapid screening of arrays of catalyst compositions.

Through exploration of the ASM binary alloy phase diagrams ${ }^{23}$ we identified 8 non-precious metals that alloy into fcc-Cu by at least 10 at.\% for some temperature below $700{ }^{\circ} \mathrm{C}$. Binary compositionally-graded thin films of each Cu-X system with compositions range from below 3 at.\% to above 18 at.\% of the alloying element were prepared by combinatorial sputtering and measured at 8 alloy compositions, creating a library of 64 alloys that were each evaluated by a series of $3 \mathrm{CVs}$ using the instrument shown in Fig. 1. The electrolyte flow at $0.16 \mathrm{~mL} \mathrm{~s}^{-1}$ through a catholyte chamber of ca. $0.32 \mathrm{~mL}$ creates a liquid-only electrochemical reactor except during sustained production of products beyond their solubility limit, e.g. prolonged partial current density above ca. $5 \mathrm{~mA} \mathrm{~cm} \mathrm{~cm}^{-2}$ for gaseous products. The electrolysis effluent flows through a pervaporator cell with poly(tetrafluoroethylene) (PTFE) membrane, which is based on the design of Clark et al., ${ }^{24}$ where the vacuum contact promotes evaporation of volatile dissolved species such as $\mathrm{H}_{2}, \mathrm{CH}_{4}$, and $\mathrm{C}_{2} \mathrm{H}_{4}$ along with a substantial amount of water. Due to the high $\mathrm{H}_{2} \mathrm{O}$ flux from the pervaporator cell, direct connection to the MS system requires substantial differential pumping as well as large background in the mass 
spectrometer signal, which prevent hydrocarbon $(\mathrm{HC})$ detection at the desired concentration and acquisition time. This issue is circumvented in the present work with an in-line molecular sieve (mol. sieve) desiccant chamber that sorbs water and alcohols to increase the partial pressure of HCs in the MS inlet by a factor of approximately 100 (Fig. S1). As a result, with $12 \mathrm{~s}$ MS measurement intervals, HCs can be detected at partial current densities $\left(J_{p}\right)$ above $30 \mu \mathrm{A} \mathrm{cm}{ }^{-2}$. The MS signals at mass-to-charge $(\mathrm{m} / \mathrm{z}$ ) values of 2,15 , and 26 provide quantitative measurement of the partial pressure of $\mathrm{H}_{2}, \mathrm{CH}_{4}$, and $\mathrm{C}_{2} \mathrm{H}_{4}$ in the post-desiccant vacuum effluent, whose signals are calibrated by saturating the flowing electrolyte with calibration gases with the cell held at open circuit. While other strategies for online product detection have enabled accelerated screening of electrocatalysts, ${ }^{22,24-26}$ we note a unique aspect of the present work is that the rapid flow and decoupling of the reactor and pervaporator ensure that products created from the entire working electrode are detected, as opposed to localized product extraction where spatial variations in mass transport, etc. can cause the measured product stream to poorly represent the electrochemical measurement, creating artifacts in key results such as FE.

Given the excellent partial current density detectability limit, the MS signals can be directly used for identifying catalysts and electrolysis settings that yield HCs. For the present purposes of identifying whether $\mathrm{Cu}$ alloys provide higher FE for HCs, especially at lower overpotentials, a CV is the desired electrochemical technique, but appreciable potential scanning rates and/or potential-dependent partial current densities result in the inability of the MS signal to reach steady state. Through modelling of the pervaporation, we find that diffusion through electrolyte in the porous PTFE membrane limits results in an exponential time constant, ca. $7 \mathrm{~s}$ for $\mathrm{H}_{2}$ and $13-14 \mathrm{~s}$ for $\mathrm{CH}_{4}$ and $\mathrm{C}_{2} \mathrm{H}_{4}, \mathrm{for}$ equilibration of the MS signal to the gas concentration in the flowing electrolyte. By calibrating model parameters with toggled flow of calibrants, we established a model for calculating the time-dependent partial current density of each product from the respective measured time-dependent MS signal, as described previously. ${ }^{27}$ For CVs at $10 \mathrm{mV} \mathrm{s}{ }^{-1}$ between un-compensated potentials of -0.4 and $-1.3 \mathrm{~V}$ vs RHE, we find that $J_{p}$ is well modelled by assuming that $J_{p}$ is the same function of applied potential during both the cathodic and anodic voltage sweeps. Furthermore, this function can be approximated by a Gaussian, which represents a peak in $J_{p}$ when the center potential of the Gaussian is within the CV limits and could also approximate an exponential function when the center potential is beyond the CV limits. Consequently, the series of MS measurements per product and per CV cycle are used to determine the corresponding 3 Gaussian parameters that minimize the loss between the modelled and measured MS signal. Fig. S2-S4 shows the stability of the measurement and of this $J_{p}$ modelling algorithm through repeated measurements on a series of Cu thin film catalysts, and Fig. 2a illustrates the measurement of $3 \mathrm{MS}$ channels and working electrode current density over 3 CV cycles with $60 \mathrm{~s}$ of open circuit potentiometry (OCP) in between to allow MS signals to return to baseline so that each CV can be modelled independently. Fig. 2 b shows the $J_{p}$ resulting from the 3-parameter Gaussian fit using a custom fitting procedure described previously. ${ }^{27}$ The MS signal corresponding to this fit is also shown in Fig. 2a. It is worth noting that the resulting $J_{p}$ is quite similar on the second and third cycles, which can differ from that of the first cycle. This variability in the first CV is expected since no electrochemical pretreatment was performed on the sputtered film and is likely related to contaminants from air exposure. As a result, the evolving product distribution during the first cycle exceeds the scope of the Gaussian-based model described above and corresponds to substantial uncertainty in the precise shape of $J_{p}$ in Fig. $2 \mathrm{~b}$. While additional modelling can be used to explore this first cycle, for the purposes of the present work we note that for all alloy catalysts the results of the second and third cycle are consistent, motivating our analysis and reporting of only the third cycle. The measured current is also smoothed with a 129-point cubic SavitskyGolay filter and then compensated for resistive losses using the measured $60 \Omega$ cell resistance. Uncompensated versions of each such figure are included in the SI.

Fig. 3 shows the MS measurements and subsequent modelling for the 8 alloy composition systems along with that of a pure Cu reference. Catalysts exhibiting current densities in excess of $5 \mathrm{~mA} \mathrm{~cm} \mathrm{~cm}^{-2}$ for a substantial fraction of each CV cycle typically causing bubble formation that gives rise to the large positive outlier values in the MS measurements. While such data points cause some uncertainty in the resulting $J_{p}$, the modelling procedure mitigates sensitivity to the outlier and preferably weights the data points at the onset of product detection. ${ }^{27}$

Complete datasets for 8 alloy systems elements are provided as Figs. S5-S12, and Fig. 3 includes the compilation of the $\mathrm{Cu}_{0.95} \mathrm{X}_{0.05}$ compositions. A range of potential-dependent $\mathrm{FE}$ signals for each product are observed, and the observed differences from the $\mathrm{Cu}$-only catalyst are quite remarkable. The figure also indicates substantial variation in the total current density, which is shown for these compositions in Fig. 4a. To further probe the composition-dependent product distributions, we consider the integral over time of this total current density as well as over each product-specific $J_{p}$, yielding electrolysis charge densities for the entire CV. The results shown in Fig. $4 \mathrm{~b}$ include a dashed line indicating the average over the Cu measurements in Fig. S3. 
To unpack this wealth of data we consider alloying elements that behave qualitatively similar and discuss in the context on the $\mathrm{Cu}$ alloy literature, which mostly involves higher concentrations of the alloying element. The lack of literature on dilute alloys of fcc-Cu is emblematic of the intuition that small concentrations of alloying elements will have no substantial impact on the catalysis, yet Fig. 4b shows that 2-3 at.\% of Sb, In or Sn is sufficient to lower total hydrocarbon production during the $\mathrm{CV}$ by a factor of more than 100, a level of sensitivity to an alloying element that to our knowledge has no precedent in electrocatalysis when the contaminant is an alloying element that is itself an electrocatalyst, as opposed to contaminants such as $\mathrm{S}$ and $\mathrm{Cl}$ that readily poison metal surfaces. ${ }^{28,29}$ It is worth noting that in the CV experiments, high partial current density for non-hydrocarbon products mitigates the ability to form hydrocarbons, not only due to the competitive concentrations of reaction intermediates, but also due to resistive losses as reflected in the range of applied potentials after resistance compensation shown in Fig. 4a.

$\mathrm{Sb}, \mathrm{In}$, and $\mathrm{Sn}$ alloys exhibit a substantial increase in the current density with the $\mathrm{FE}$ for $\mathrm{H}_{2}$ being relatively unchanged, indicating that the suppression in hydrocarbon formation is the result of a substantially increased activity for other products, perhaps the $2 \mathrm{e}^{-}$products that these main group catalysts are known to form in the absence of Cu. These results are in agreement with previous reports on $\mathrm{Cu}-\mathrm{In}^{9-11}$ and $\mathrm{Cu}-\mathrm{Sn}^{14}$ catalysts where the weak $\mathrm{H}$ adsorption and strong $\mathrm{O}$ binding of $\mathrm{In}$ and $\mathrm{Sn}$ enhances selectivity toward formate and $\mathrm{CO}$. For example, FE of $90 \%$ for formate with $\mathrm{Cu}$ $\mathrm{Sn}$ and for $\mathrm{CO}$ with $\mathrm{Cu}$-In were reported at low potentials, with the present work being the first to explore more dilute alloys of these elements. While further study of the highly active $\mathrm{Cu}-(\mathrm{Sb}, \mathrm{In}, \mathrm{Sn})$ catalysts may be of interest for formation of products other than $\mathrm{HCs}$, their primary scientific importance for $\mathrm{CO}_{2} \mathrm{RR}$ is understanding how such a small concentration can so strongly influence product distribution. In the concerted community effort to understand the uniqueness of $\mathrm{Cu}$, these alloys provide a new line of inquiry.

The $\mathrm{Cu}-\mathrm{Zn}$ alloys share some important similarities and differences compared to the $\mathrm{Sb}$, In, and Sn systems. The hydrocarbon formation is less sensitive to alloy concentration in the 2-6 at.\% range. Catalysts with higher $\mathrm{Zn}$ concentrations exhibit the highest $\mathrm{H}_{2}$ production among all the alloys, which not only hampers formation of hydrocarbon but also other $\mathrm{CO}_{2} \mathrm{RR}$ products. These results are somewhat surprising given literature precedent of $\mathrm{Cu}-\mathrm{Zn}$ catalysts. Zn-rich alloy films ${ }^{30}$ and nanoporous $\mathrm{Zn}$-rich structures ${ }^{18}$ have been studied for formate and CO production at low overpotential, which are not directly comparable to the $\mathrm{Zn}$-poor catalysts in Fig. 4 and collectively indicate that product distributions of $\mathrm{Cu}-\mathrm{Zn}$ catalysts do not vary monotonically with composition. $\mathrm{Zn}$ coatings on $\mathrm{Cu}$ have been shown to suppress HER and increase $\mathrm{CH}_{4}$ production, ${ }^{31}$ in stark contrast to the results of Fig. 4, indicating that the surface and near-surface $\mathrm{Zn}$ in the alloy catalysts of the present work are critically different from the previously reported application of Zn coatings. Ren et al. ${ }^{32}$ studied electrodeposited Cu-Zn alloys with Zn concentrations of 9-30 at.\% and focused on the increased ethanol production at high Zn concentrations. They also observed increased total current with $\mathrm{Cu}-\mathrm{Zn}$ alloys compared to $\mathrm{Cu}$ at potentials above $-0.95 \mathrm{~V}$ vs RHE, in agreement with the present work, although the most $\mathrm{Cu}$-rich alloy in that work ( 9 at.\% $\mathrm{Zn}$ ) exhibited an increase in $\mathrm{FE}$ of $\mathrm{C}_{2} \mathrm{H}_{5} \mathrm{OH}$, which is not measured by our study. Feng et al. ${ }^{17}$ observed enhanced $\mathrm{FE}$ for $\mathrm{C}_{2} \mathrm{H}_{4}$ on oxide-derived $\mathrm{Cu}-\mathrm{Zn}$ nanoparticles where $\mathrm{Zn}$ concentrations from 12 to 50 at.\% were studied, resulting in identification of 20 at.\% $\mathrm{Zn}$ as optimal for $\mathrm{C}_{2} \mathrm{H}_{4}$ formation. In this context, the results of Fig. 4 indicate that the morphology and exposed catalyst sites from the oxide-derived synthesis technique is more critical than the presence of $\mathrm{Zn}$, which is commensurate with recent studies ${ }^{33}$ on product-specific active sites in oxide-derived $\mathrm{Cu}$ catalysts and motivates further inspection of whether $\mathrm{Zn}$ directly plays a catalytic role in increasing the $\mathrm{FE}$ of $\mathrm{C}_{2} \mathrm{H}_{4}$ or if it instead stabilizes the hypothesized unique $\mathrm{Cu}$ sites generated through electrochemical reduction from the metal oxide.

While the 2 at.\% Co alloy enhances hydrocarbon formation by a factor of about 2, Co is otherwise the most innocuous alloying element with no substantial change in the overall current and slightly increasing $\mathrm{H}_{2}$ and decreasing $\mathrm{HC}$ production with increasing alloying concentration. Similar trends of hydrocarbon formation over CuCo was observed by Grote and his coworkers. ${ }^{22} \mathrm{Al}$ is also somewhat innocuous with the primary effect being increase $\mathrm{H}_{2}$ formation, which is markedly different from the heavier main group elements discussed above.

Other than the overall sensitivity of $\mathrm{Cu}$ to small alloying concentrations, perhaps the most intriguing observation is the select cases where the alloying element changes the relative production of $\mathrm{CH}_{4}$ and $\mathrm{C}_{2} \mathrm{H}_{4}$, which is shown in Fig. $\mathrm{S} 13$. The most $\mathrm{Cu}$-rich alloys with $\mathrm{Zn}, \mathrm{Al}$, and $\mathrm{Co}$ slightly increase selectivity for $\mathrm{CH}_{4}$. The 2-5 at.\% $\mathrm{Sb}$ catalysts are interesting in that they eliminate $\mathrm{CH}_{4}$ from detectability but not $\mathrm{C}_{2} \mathrm{H}_{4}$, posing the possibility that improved catalysts could be obtained if this selectivity control could be harnessed without the increased FE in other products. A similar but more gradual trend is observed with $\mathrm{Ni}$ and $\mathrm{Mn}$, with both showing a consistent increase in relative $\mathrm{C}_{2} \mathrm{H}_{4}$ production with increasing alloy concentration. While the total hydrocarbon production of $\mathrm{Ni}$ also declines with increasing alloy concentration, the $\mathrm{Mn}$ alloys up to 5 at.\% show slightly higher total hydrocarbon production, with the 3-4 at.\% Mn 
increasing the fraction of $C_{2}$ hydrocarbons (compared to total hydrocarbons) from the ca. 40 at.\% value observed with pure Cu to ca. 60 at.\%.

The alloys with 5 at.\% CuSn and CuMn were measured with XPS (Fig S14) to assess whether the near-surface concentrations of the alloying elements far exceeded the bulk values, and while enhancements of $1.6 \times$ and $2.8 \times$, respectively, were observed, the sensitivity of product distribution to alloy concentration remains remarkable and largely unexplained by present understanding of $\mathrm{CO}_{2} \mathrm{RR}$ on $\mathrm{Cu}$-based catalysts. Theoretical characterization of absorption energies for bimetallic alloys ${ }^{34}$ will help elucidate the composition-selectivity trends, although such data is not presently available in the dilute alloy concentration range and will additionally need to consider elemental surface segregation and reorganization processes. Since it is unlikely that such dilute alloys substantially alter the electronic structure of $\mathrm{Cu}$, the most likely underlying phenomenon is that the hydrocarbon-forming $\mathrm{Cu}$ sites have a relatively high surface energy that concomitantly drives surface alloying elements to these same sites, enabling a dilute concentration of these active sites to be contaminated by dilute alloy concentrations. Validating this hypothesis will require further experimentation and/or theoretical investigation, and the notable exceptions to this trend, such as the $C_{2}$ product selectivity of $\mathrm{Mn}$, merit further investigation. These results also represent a cautionary tale for development of $\mathrm{CO}_{2} \mathrm{RR}$ electrolysis devices as any plating of metal contaminants in the electrolyte can substantially alter product distributions, creating demanding requirements for elimination of trace metal contaminants. A notable precedence for this level of sensitivity to trace metals exists in alkaline oxygen evolution electrocatalysis where trace adventitious Fe from the electrolyte substantially influenced catalytic activity for initially Fe-free catalysts. ${ }^{35}$ While the activity boost in that situation posed problems for scientific studies but not electrode development, the sensitivity of Cu to metal impurities poses more substantial challenges for electroctalayst research and development.

\section{Conclusions}

We address challenges in $\mathrm{CO}_{2}$ reduction electrcatalyst discovery through the design of an electrochemical flow cell with online mass spectroscopy-based quantification of the Faradaic efficiency for $\mathrm{H}_{2}, \mathrm{CH}_{4}$, and $\mathrm{C}_{2} \mathrm{H}_{4}$ with $12 \mathrm{~s}$ measurement intervals and partial current detectability of ca. $0.03 \mathrm{~mA} \mathrm{~cm}^{-2}$, enabling high throughput screening of Cu bimetallic alloys with $\mathrm{Al}, \mathrm{Mn}, \mathrm{Co}, \mathrm{Ni}, \mathrm{Zn}, \mathrm{In}, \mathrm{Sn}$, and $\mathrm{Sb}$. The product distribution of Cu-rich catalysts is remarkably sensitive to alloy concentration with 2 at.\% of some elements causing tenfold decrease of hydrocarbon formation, indicating that alloying elements segregate to critical sites for hydrocarbon formation, which poses substantial challenges for development and sustainable operation of Cu-based catalysts.

\section{Conflicts of interest}

There are no conflicts to declare.

\section{Notes and references}

1 P. D. Luna, C. Hahn, D. Higgins, S. A. Jaffer, T. F. Jaramillo and E. H. Sargent, Science, 2019, 364, eaav3506.

2 Y. Hori, H. Wakebe, T. Tsukamoto and O. Koga, Electrochimica Acta, 1994, 39, 1833-1839.

3 K. P. Kuhl, E. R. Cave, D. N. Abram and T. F. Jaramillo, Energy \& Environmental Science, 2012, 5, 7050-7059.

4 S. Nitopi, E. Bertheussen, S. B. Scott, X. Liu, A. K. Engstfeld, S. Horch, B. Seger, I. E. L. Stephens, K. Chan, C. Hahn, J. K. Nørskov, T. F. Jaramillo and I. Chorkendorff, Chemical Reviews, 2019, 119, 7610-7672.

5 K. P. Kuhl, T. Hatsukade, E. R. Cave, D. N. Abram, J. Kibsgaard and T. F. Jaramillo, Journal of the American Chemical Society, 2014, 136, 14107-14113.

6 A. A. Peterson and J. K. Nørskov, The Journal of Physical Chemistry Letters, 2012, 3, 251-258.

7 A. Vasileff, C. Xu, Y. Jiao, Y. Zheng and S.-Z. Qiao, Chem.

8 J. Christophe and T. Doneux, 2012, 139-146.

9 A. Jedidi, S. Rasul, D. Masih, L. Cavallo and K. Takanabe, 2015, 19085-19092. 
Z. B. Ho, T. S. Gray, K. B. Moraveck, T. B. Gunnoe and G. Zangari, , DOI:10.1021/acscatal.7b01161.

A. J. Mart, S. Mitchell, R. Hauert and J. Pe, 2016, 2-11.

12 S. Rasul, D. H. Anjum, A. Jedidi, Y. Minenkov, L. Cavallo and K. Takanabe, Angewandte Chemie - International Edition, 2015, 54, 2146-2150.

13 J. He, K. E. Dettelbach, D. A. Salvatore, T. Li and C. P. Berlinguette, 2017, 6068-6072.

14 M. Morimoto, Y. Takatsuji, R. Yamasaki, H. Hashimoto, I. Nakata, T. Sakakura and T. Haruyama, Electrocatalysis, 2018, 9, 323-332.

15 M. Li, J. Wang, P. Li, K. Chang, C. Li, T. Wang and B. Jiang, 2016, 4776-4782.

16 D. Ren, B. S.-H. Ang and B. S. Yeo, ACS Catal., 2016, 6, 8239-8247.

17 Y. Feng, Z. Li, H. Liu, C. Dong, J. Wang, S. A. Kulinich and X. Du, Langmuir, 2018, 34, 13544-13549.

18 H. Hu, Y. Tang, Q. Hu, P. Wan, L. Dai and X. J. Yang, Applied Surface Science, 2018, 445, 281-286.

19 G. Yin, H. Abe, R. Kodiyath, S. Ueda, N. Srinivasan, A. Yamaguchi and M. Miyauchi, J. Mater. Chem. A, 2017, 5, 1211312119.

20 G. Keerthiga and R. Chetty, J. Electrochem. Soc., 2017, 164, H164-H169.

21 E. L. Clark, C. Hahn, T. F. Jaramillo and A. T. Bell, 2017, 15848-15857.

22 J. P. Grote, A. R. Zeradjanin, S. Cherevko, A. Savan, B. Breitbach, A. Ludwig and K. J. J. Mayrhofer, Journal of Catalysis, 2016, 343, 248-256.

23 ASM Alloy Phase Diagram Database ${ }^{\mathrm{TM}}$ - ASM International, https://www.asminternational.org/home//journal_content/56/10192/15469013/DATABASE, (accessed June 25, 2019).

24 12119.

E. L. Clark, M. R. Singh, Y. Kwon and A. T. Bell, Analytical Chemistry, 2015, 87, 8013-8020.

E. L. Clark and A. T. Bell, , DOI:10.1021/jacs.8b04058.

K. Jambunathan, S. Jayaraman and A. C. Hillier, Langmuir, 2004, 20, 1856-1863.

Y. Lai, R. Jones, Y. Wang, L. Zhou and J. Gregoire, submitted, see supporting document.

A. F. Ghenciu, Current Opinion in Solid State and Materials Science, 2002, 11.

N. Job, M. Chatenet, S. Berthon-Fabry, S. Hermans and F. Maillard, Journal of Power Sources, 2013, 240, $294-305$.

G. Yin, H. Abe, R. Kodiyath, S. Ueda, N. Srinivasan, A. Yamaguchi and M. Miyauchi, J. Mater. Chem. A, 2017, 5, 12113-

G. Keerthiga and R. Chetty, J. Electrochem. Soc., 2017, 164, H164-H169.

D. Ren, B. S.-H. Ang and B. S. Yeo, ACS Catal., 2016, 6, 8239-8247.

Y. Lum and J. W. Ager, Nature Catalysis, 2019, 2, 86.

O. Mamun, K. T. Winther, J. R. Boes and T. Bligaard, Scientific Data, 2019, 6, DOI:10.1038/s41597-019-0080-z.

L. Trotochaud, S. L. Young, J. K. Ranney and S. W. Boettcher, J. Am. Chem. Soc., 2014, 136, 6744-6753. 


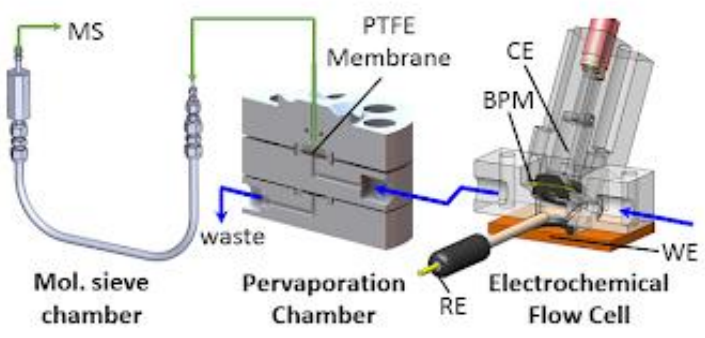

Figure 1: Electrolyte (blue) and in-vacuum product stream (green) flows through the 3 components between the electrolyte reservoir and MS detector. The planar working electrode is pressed against the o-ring at the bottom of the electrochemical cell. The inlet flow tube is directed toward the working electrode (WE), and the outlet flow tube terminates near the membrane at the top of the cell so that any bubbles are readily removed. The reference electrode (RE) is horizontal, terminating near the center of the chamber, ca. $2.8 \mathrm{~mm}$ above the WE, and the counter electrode (CE) is separated by the bipolar membrane (BPM). The electrolyte is then injected in the center of a cylindrical pervaporation chamber with volatile species crossing the PTFE membrane, creating a product stream that is differentially pumped by the mol. sieve before continuous injection in the MS for product quantification.

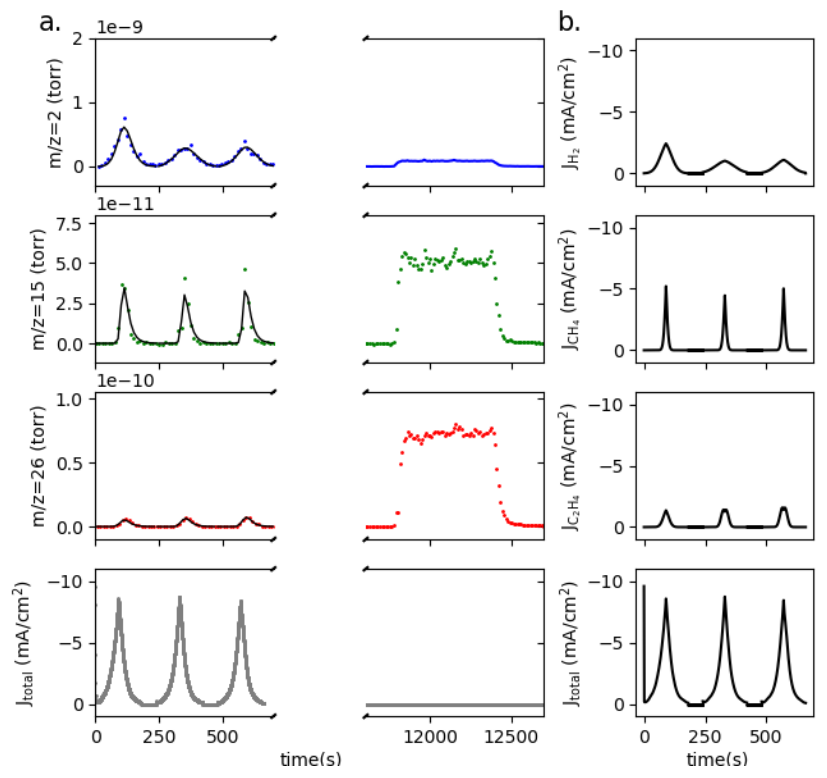

Figure 2. EC-MS demonstration with a thin film Cu electrode. a) Typical experimental process for catalyst screening including $3 \mathrm{CV}$ cycles separated by OCP for enabling MS to return to baseline. After performing such measurements on a series of catalysts, a daily calibration is performed by flowing through electrolyte bubbled with calibration gas containing $0.4 \% \mathrm{H}_{2}, 0.4 \% \mathrm{CH}_{4}$, and $0.4 \% \mathrm{C}_{2} \mathrm{H}_{4}$ in $\mathrm{CO}_{2}$. b) $U$ sing this calibration and pervaporation model, the modeled partial current density for each species is calculated and compared to the measured total current. 

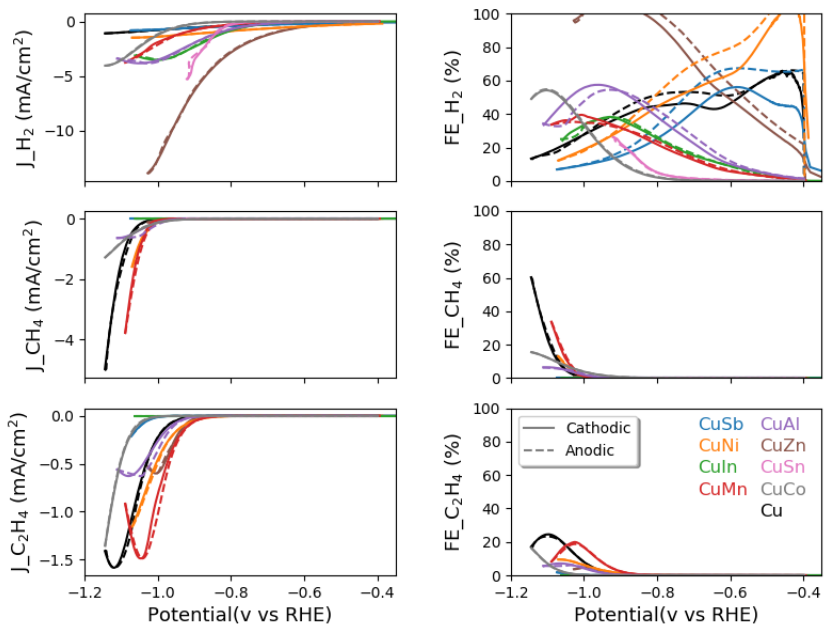

Figure 3. The $J_{p}$ (left column) and FE (right column) for the third CV cycle is shown for each of the 8 Cu-based bimetallic catalysts with ca. $5 \%$ of the alloying metal. The potentials include resistance compensation and legends in bottom-right panel apply to all 6 panels.
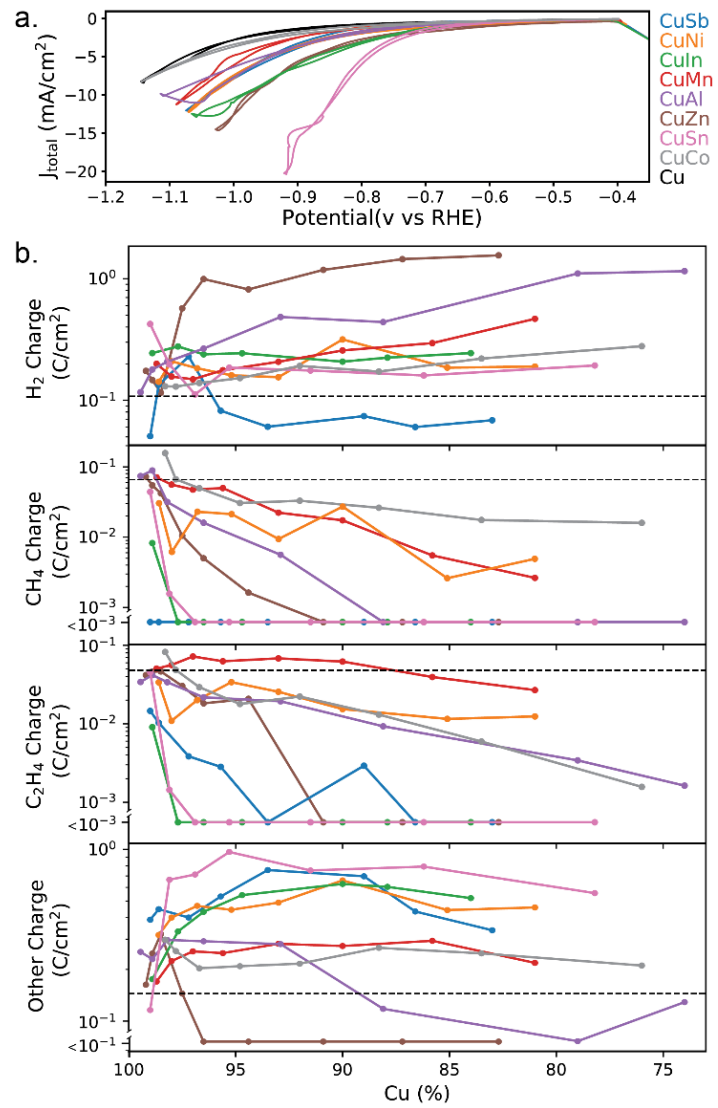

Figure 4. Charge density (integral of $J_{p}$ over time(s)) for the third CV cycle is shown for each of the 8 alloy elements as a function of alloy concentration. The dashed black line indicates the average charge density for pure $\mathrm{Cu}$. The bottom 3 panels include a broken charge density axis corresponding to product generation near or below the detectability limit. 